\title{
ESTIMATES FOR BEST APPROXIMATION TO RATIONAL FUNCTIONS $\left(^{1}\right)$
}

\author{
BY
}

\section{S. J. POREDA}

\begin{abstract}
Estimates for the deviation of certain rational functions and their polynomials of best uniform approximation on various sets are given. As a result, in some cases these deviation and polynomials are explicitly calculated. For example, the polynomials of best uniform approximation to the function $(\alpha z+\beta) /(z-a)(1-\bar{a} z)$, $|a| \neq 1$, on the unit circle are given.
\end{abstract}

1. Introduction. For a complex-valued function $f$, continuous on a compact set $E$ in the plane, let $p_{n}(f, E)$ denote the polynomial of degree $n, n \in Z^{+}$, of best uniform approximation to $f$ on $E$ and, let $\rho_{n}(f, E)=\max _{z \in E}\left|f(z)-p_{n}(f, E)(z)\right|$. Also, let $U$ denote the unit circle $|z|=1$.

$p_{n}(f, E)$ and $\rho_{n}(f, E)$ are known only for a small class of functions and sets, and in particular, if $f$ is a rational function and not a polynomial, the only relatively nontrivial results in this direction have been given by $\mathrm{P}$. Chebyshev [1, p. 58] who calculated $p_{n}(1 /(x-a),[-1,1])$, for $a>1$ or $a<-1$, and by $\mathrm{S}$. $\mathrm{Al}^{\prime}$ per [2] who calculated $p_{n}(1 /(z-a), U)$ for $|a|>1$.

In what follows, the author finds estimates for the deviation $\rho_{n}(f, E)$ for several classes of rational functions and sets, and using these estimates, $p_{n}(f, E)$ is calculated for a class of rational functions and sets thereby generalizing the above mentioned result of $\mathrm{Al}^{\prime}$ per.

2. Preliminary results. We begin by stating $\mathrm{Al}^{\prime}$ per's result.

THEOREM 1 [2]. For $n \in Z^{+}$, and $|a|>1$,

$$
p_{n}(1 /(z-a), U)=\left[1-K z^{n}(1-\bar{a} z)\right] /(z-a), \text { where } K=\left[a^{n}\left(1-|a|^{2}\right)\right]^{-1} \text {. }
$$

If $|a|<1, p_{n}(1 /(z-a), U)$ can also be found, in fact it is a constant for all $n$.

THEOREM 2. For $n \in Z^{+}$and $|a|<1$,

$$
p_{n}(1 /(z-a), U)=[1-K(1-\bar{a} z)] /(z-a), \text { where } K=\left(1-|a|^{2}\right)^{-1} \text {. }
$$

Proof. By letting $w=1 / z$ the problem of finding $p_{n}(1 /(z-a), U),|a|<1$, can be shown to be equivalent to finding $p_{n}\left(w^{n+1} /(1-a w), U\right)$ which can be calculated by applying Theorem 1 to yield the indicated result.

Received by the editors June 26, 1970.

AMS 1969 subject classifications. Primary 4140; Secondary 3070, 4141.

Key words and phrases. Best uniform approximation, rational function, lemniscate.

( $\left.{ }^{1}\right)$ This represents a portion of the author's doctoral dissertation, directed by Professor Mishael Zedek, at the University of Maryland.

Copyright (C) 1971, American Mathematical Society 
3. An estimate for $\rho_{n}(f, U)$. Since $U$ will be the set we will be mainly concerned with, set $p_{n}(f, U)=p_{n}(f)$, and $\rho_{n}(f, U)=\rho_{n}(f)$. We consider here a rational function $f(z)$ of the form $f(z)=g(z)+h(z)$, where $g(z)=\alpha /(z-a), h(z)=\beta /(1-\bar{b} z),|a|>1$, $|b|>1$, and where in addition, either $a$ and $b$ lie on the same ray from the origin or $|a|$ and $|b|$ are sufficiently large, e.g. $|a|>9$ and $|b|>9$. Using Theorems 1 and 2, we can calculate $\rho_{n}(g)$ and $\rho_{n}(h)$, which will give us an upper bound for $\rho_{n}(f)$ since $\rho_{n}(f) \leqq \rho_{n}(g)+\rho_{n}(h)$. To obtain a lower bound we start by defining

$$
\phi(z)=[(1-\bar{a} z)(z-b)(1-a)(1-\bar{b}) /(z-a)(1-\bar{b} z)(1-\bar{a})(1-b)]^{1 / 2},
$$

where $\pi \geqq \arg w^{1 / 2}>-\pi$. Now if we let $\theta_{0}=\max _{z \in U}\left|\arg ^{*} \phi(z)\right|$, where $\pi \geqq \arg * w$ $>-\pi$, we have, using the geometric restrictions placed on $a$ and $b$, that $0 \leqq \theta_{0}<\pi / 2$, and the following:

THEOREM 3.

$$
\begin{aligned}
{\left[\rho_{n}(\alpha /(z-a))+\rho_{n}(\beta /(1-\bar{b} z))\right] \cos \theta_{0} } & \leqq \rho_{n}(\alpha /(z-a)+\beta /(1-\bar{b} z)) \\
& \leqq\left[\rho_{n}(\alpha /(z-a))+\rho_{n}(\beta /(1-\bar{b} z))\right] .
\end{aligned}
$$

Before proving the above we note that if $\theta_{0}=0$, or equivalently, if $a=b$, we have

$$
\rho_{n}(\alpha /(z-a)+\beta /(1-\bar{a} z))=\rho_{n}(\alpha /(z-a))+\rho_{n}(\beta /(1-\bar{a} z)) .
$$

4. Examples of best approximation. Using the special case, mentioned above, of Theorem 3, we can now explicitly calculate $p_{n}((c z+d) /(z-a)(1-\bar{a} z))$.

Corollary 1. For $n \in Z^{+}$and $|a|>1$,

$$
p_{n}((c z+d) /(z-a)(1-\bar{a} z))=\left[c z+d-K_{1} z^{n}(1-\bar{a} z)^{2}-K_{2}(z-a)^{2}\right] /(z-a)(1-\bar{a} z),
$$

where $K_{1}=(c a+d) / a^{n}\left(1-|a|^{2}\right)^{2}$ and $K_{2}=\bar{a}(c+d \bar{a}) /\left(1-|a|^{2}\right)^{2}$.

It should be noted that, by choosing appropriate values for $c$ and $d$ in Corollary 1 , we have Theorems 1 and 2 as special cases. We will now give a more generalized version of Corollary 1 in which the sets under consideration are lemniscates. Of course, the unit circle $U$ is itself a lemniscate.

TheOREM 4. For $n \in Z^{+},|a|>1, p(z)$ a polynomial of degree $\lambda, \lambda \geqq 1$, and $E$ the lemniscate $\{|p(z)|=1\}$,

$$
\begin{aligned}
p_{n}((c p(z)+d) /(p(z)-a)(1-\bar{a} p(z)), E) \\
=\left[c p(z)+d-L_{1} p(z)^{k}(1-\bar{a} p(z))^{2}-L_{2}(p(z)-a)^{2}\right] /(p(z)-a)(1-\bar{a} p(z)),
\end{aligned}
$$

where $L_{1}=(c a+d) / a^{k}\left(1-|a|^{2}\right)^{2}, L_{2}=\bar{a}(c+d \bar{a}) /\left(1-|a|^{2}\right)^{2}$, and $\lambda k \leqq n<\lambda(k+1)$.

In order to prove the above we need use of the fact that the polynomials of best approximation to a continuous function of $p(z)$ on $E$ are polynomials in $p(z)$, that is, they contain only powers of $p(z)$. Then Theorem 4 will follow directly from Corollary 1. 
LEMMA 1. Let $G(z)$ be a continuous function of $p(z)$ defined on $E$, i.e., if $p\left(z_{1}\right)$ $=p\left(z_{2}\right)$ then $G\left(z_{1}\right)=G\left(z_{2}\right)$. Then $p_{n}(G, E)(z)$ is a polynomial in $p(z)$.

Proof. Let $\Psi_{i}: E \rightarrow E$, for $i=1,2, \ldots, \lambda$, be functions which have the property that if $z_{0} \in E$ then

$$
p(z)-p\left(z_{0}\right)=a_{\lambda} \sum_{i=1}^{i=\lambda}\left(z-\Psi_{i}\left(z_{0}\right)\right)
$$

where $a_{\lambda}$ is the leading coefficient of $p(z)$. The existence of such a set of functions is guaranteed by the Fundamental Theorem of Algebra.

Furthermore, by Newton's Formula we have that, for $1 \leqq j \leqq \lambda-1$,

$$
\frac{1}{\lambda} \sum_{i=1}^{i=\lambda}\left[\Psi_{i}(z)\right]^{j}=B_{j} \quad \text { for all } z \in E,
$$

where $B_{j}$ is a constant independent of $z$.

Now since $p(z)=p\left(\Psi_{i}(z)\right)$ for $z \in E$ and $i=1,2, \ldots, \lambda$, we also have that $G(z)$ $=G\left(\Psi_{i}(z)\right)$. So letting $p_{n}=p_{n}(G, E)$ we have that, for $i=1,2, \ldots, \lambda$,

$$
\left\|G(z)-p_{n}(z)\right\|_{E} \geqq\left\|G\left(\Psi_{i}(z)\right)-p_{n}\left(\Psi_{i}(z)\right)\right\|_{E}=\left\|G(z)-p_{n}\left(\Psi_{i}(z)\right)\right\|_{E} .
$$

Now let $P_{n}(z)=(1 / \lambda) \sum_{i=1}^{i=\lambda} p_{n}\left(\Psi_{i}(z)\right)$. Using the triangle inequality we have

$$
\left\|G(z)-P_{n}(z)\right\|_{E} \leqq \frac{1}{\lambda} \sum_{i=1}^{i=\lambda}\left\|G(z)-p_{n}\left(\Psi_{i}(z)\right)\right\|_{E}
$$

and now using (4.2) we can write

$$
\left\|G(z)-P_{n}(z)\right\|_{E} \leqq\left\|G(z)-p_{n}(z)\right\|_{E} .
$$

Now since $p_{n}(z)$ and $p(z)$ are polynomials of respective degrees $n$ and $\lambda$, we may write $p_{n}(z)$ in the form

$$
p_{n}(z)=Q_{0}(p(z))+z Q_{1}(p(z))+\cdots+z^{\lambda-1} Q_{\lambda-1}(p(z)),
$$

where $k \lambda \leqq n<(k+1) \lambda$ and where $Q_{i}$ is a polynomial of degree $k$ for $i=0,1, \ldots$, $\lambda-1$. Thus $P_{n}(z)$ becomes

$$
\begin{aligned}
P_{n}(z)=Q_{0}(p(z)) & +\frac{1}{\lambda}\left[\sum_{i=1}^{i=\lambda} \Psi_{i}(z)\right] Q_{1}(p(z))+\cdots \\
& +\frac{1}{\lambda}\left[\sum_{i=1}^{i=\lambda}\left[\Psi_{i}(z)\right]^{\lambda-1}\right] Q_{\lambda-1}(p(z)) .
\end{aligned}
$$

But then by (4.1) we have that

$$
P_{n}(z)=Q_{0}(p(z))+B_{1} Q_{1}(p(z))+\cdots+B_{\lambda-1} Q_{\lambda-1}(p(z)) .
$$

Thus $P_{n}(z)$ is a polynomial, and in particular a polynomial in $p(z)$, so by (4.3) and the uniqueness of $p_{n}(G, E)$ we have that $p_{n}(z)=P_{n}(z)$. 
Theorem 4 can be made even more general if we consider functions of the form $g(z)=p(z)^{s}\left(\alpha p(z)^{t}+\beta\right) /\left(1-a p(z)^{t}\right)\left(p(z)^{t}-a\right)$ on $E$ where $a, p(z), E$ are as before and where $t$ and $s$ are integers with $1 \leqq s<t$. Then if we let

$$
h(z)=\left(\alpha p(z)^{t}+\beta\right) /\left(1-a p(z)^{t}\right)\left(p(z)^{t}-a\right)
$$

we get that $p_{n}(g, E)(z)=p(z)^{s} p_{M}(h, E)(z)$ where $M=m t \lambda$ and where $(m t+s) \lambda$ $\leqq n<((m+1) t+s) \lambda$. Using Theorem 4 we can find $p_{M}(h, E)$ and thus we can also find $p_{n}(g, E)$.

5. Proof of Theorem 3. Before proceeding we will give the proof of Theorem 3, which in itself is quite interesting.

Proof. We first consider the case where the polynomial is of even degree or where $n=2 m$. Let $f(z), g(z), h(z)$ be as before.

Set $q_{2 m}(z)=p_{2 m}(g)(z)+p_{2 m}(h)(z), \rho_{1}=\rho_{2 m}(g)$ and $\rho_{2}=\rho_{2 m}(h)$. Then

$$
\begin{aligned}
f(z)-q_{2 m}(z) & =g(z)-p_{2 m}(g)(z)+h(z)-p_{2 m}(h)(z) \\
& =\mu_{1} \rho_{1} z^{2 m}(1-\bar{a} z) /(z-a)+\mu_{2} \rho_{2}(z-b) /(1-\bar{b} z) \\
& =z^{m} \phi(z)\left[K_{1} z^{m}(1-\bar{a} z) / \phi(z)(z-a)+K_{2} \phi(z)(z-a) / z^{m}(1-\bar{a} z)\right],
\end{aligned}
$$

where $\left|\mu_{1}\right|=\left|\mu_{2}\right|=1, K_{1}=\mu_{1} \rho_{1}$ and $K_{2}=\mu_{2} \rho_{2}(1-\bar{a})(1-b) /(1-a)(1-\bar{b})$.

Since the function $\Psi(z)=z^{m}(1-\bar{a} z) / \phi(z)(z-a)$ maps $U$ onto itself $m+1$ times, the function $\left[K_{1} \Psi(z)+K_{2} / \Psi(z)\right]$ maps $U$ onto an ellipse centered at the origin $m+1$ times, and so its maximum modulus on $U$ is attained at the $2 m+2$ points where $[\Psi(z)]^{2}=\left(K_{2} / K_{1}\right) /\left|K_{2} / K_{1}\right|$. Let $\left\{z_{i}\right\}_{i=1}^{2 m+2}$ denote these points and label them in such a way that $0 \leqq \arg z_{1}<\arg z_{2}<\cdots<\arg z_{2 m+2} \leqq 2 \pi$ and

$$
K_{1} \Psi\left(z_{k}\right)+K_{2} / \Psi\left(z_{k}\right)=(-1)^{k+1} K_{0} \quad \text { for } k=1,2, \ldots, 2 m+2,
$$

where $\left|K_{0}\right|=\left|K_{1}\right|+\left|K_{2}\right|=\rho_{1}+\rho_{2}$.

Now let $r_{2 m}(z)=p_{2 m}(f)(z)-q_{2 m}(z)$, and $\varepsilon=\rho_{1}+\rho_{2}-\rho_{2 m}(f),\left(0 \leqq \varepsilon \leqq \rho_{1}+\rho_{2}\right)$. Then

$$
\begin{aligned}
\left|f\left(z_{k}\right)-p_{2 m}(f)\left(z_{k}\right)\right| & =\left|\left[f\left(z_{k}\right)-q_{2 m}\left(z_{k}\right)\right] / z_{k}^{m}-r_{2 m}\left(z_{k}\right) / z_{k}^{m}\right| \\
& =\left|\phi\left(z_{k}\right)\left[K_{1} \Psi\left(z_{k}\right)+K_{2} / \Psi\left(z_{k}\right)\right]-r_{2 m}\left(z_{k}\right) / z_{k}^{m}\right| \\
& =\left|\phi\left(z_{k}\right)(-1)^{k+1} K_{0}-r_{2 m}\left(z_{k}\right) / z_{k}^{m}\right| \leqq \rho_{1}+\rho_{2}-\varepsilon,
\end{aligned}
$$

for $k=1,2, \ldots, 2 m+2$. Thus we see that $r_{2 m}\left(z_{k}\right) / K_{0} z_{k}^{m}$ is contained in the disc

$$
\left|w-(-1)^{k+1} \phi\left(z_{k}\right)\right| \leqq\left(\rho_{1}+\rho_{2}-\varepsilon\right) /\left(\rho_{1}+\rho_{2}\right), \quad \text { for } k=1,2, \ldots, 2 m+2 .
$$

Letting $\eta=\operatorname{Arcsin}\left[\left(\rho_{1}+\rho_{2}-\varepsilon\right) /\left(\rho_{1}+\rho_{2}\right)\right]$ and $\theta_{0}$ as before, we can see that if $\theta_{0}+\eta \leqq \pi / 2$, then $r_{2 m}\left(z_{k}\right) / K_{0} z_{k}^{m}$ is in the half plane $(-1)^{k+1} \operatorname{Re} w \geqq 0$ for $1 \leqq k \leqq 2 m+2$. This is impossible since it implies that the trigonometric polynomial of degree $m, \operatorname{Re}\left[r_{2 m}(z) / K_{0} z^{m}\right]$, has $2 m+2$ zeros on $U$. Thus, we must have $\theta_{0}+\eta>\pi / 2$, or $\operatorname{Arcsin}\left[\left(\rho_{1}+\rho_{2}-\varepsilon\right) /\left(\rho_{1}+\rho_{2}\right)\right]>\pi / 2-\theta_{0}$. Thus it follows that

$$
\rho_{1}+\rho_{2}-\varepsilon>\left(\rho_{1}+\rho_{2}\right) \sin \left(\pi / 2-\theta_{0}\right)=\left(\rho_{1}+\rho_{2}\right) \cos \theta_{0} .
$$


Now if $n$ is odd, consider instead the function $F(z)=G(z)+H(z)$ where $G(z)=g\left(z^{2}\right)$ and $H(z)=h\left(z^{2}\right)$. Replacing $z$ by $z^{2}$ in the foregoing argument yields

$$
\left(\rho_{2 n}(G)+\rho_{2 n}(H)\right) \cos \theta_{0} \leqq \rho_{2 n}(F),
$$

and by applying Lemma 1 , we can show that

$$
\rho_{2 n}(G)=\rho_{n}(g), \quad \rho_{2 n}(H)=\rho_{n}(h) \quad \text { and } \quad \rho_{2 n}(F)=\rho_{n}(f) .
$$

Thus the proof of our theorem is complete.

6. Other estimates for $\rho_{n}(f, U)$. We now give another technique for estimating $\rho_{n}(f, U)$ for some rational functions $f$. Although we consider only rational functions having two poles, this technique has wider applications, which we omit, since they simply constitute repeated applications of the arguments given below.

By Theorem 4, we know that if $|a|>1$ then $\rho_{n}(\alpha /(z-a))=\left|\alpha / a^{n}\left(|a|^{2}-1\right)\right|$ and if $|b|<1$ then $\rho_{n}(\beta /(z-b))=\left|\beta /\left(1-|b|^{2}\right)\right|$. The following theorem permits us to estimate the quantity $\rho_{n}(\alpha /(z-a)+\beta /(z-b))$.

\section{TheOREM 5.}

$$
\begin{aligned}
|(a-b) /(1-\bar{b} a)| \rho_{n}(\alpha /(z-a)) & \leqq \rho_{n}(\alpha /(z-a)+\beta /(z-b)) \\
& \leqq \rho_{n}(\alpha /(z-a))+\rho_{n}(\beta /(z-b)) \text { if }|a|>1,|b|>1,
\end{aligned}
$$

and

$$
\begin{aligned}
\rho_{n}(\beta /(z-b)) & \leqq \rho_{n}(\alpha /(z-a)+\beta /(z-b)) \\
& \leqq \rho_{n}(\alpha /(z-a))+\rho_{n}(\beta /(z-b)) \text { if }|a|>1 \text { and }|b|<1 .
\end{aligned}
$$

In case (1), we have equality on the left if

$$
\beta=-\alpha b^{n}(1-\bar{a} b)\left(1-|b|^{2}\right) / a^{n}(1-\bar{b} a)\left(1-|a|^{2}\right) .
$$

In case (2), we note that $\rho_{n}(\alpha /(z-a)+\beta /(z-b))$ converges to $\rho_{n}(\beta /(z-b))$ $=\left|\beta /\left(1-|b|^{2}\right)\right|$ as $n$ increases, since $\rho_{n}(\alpha /(z-a))=\left|\alpha / a^{n}\left(|a|^{2}-1\right)\right|$ converges to zero as $n$ increases.

We now consider rational functions having a pole of multiplicity two at a point outside $U$.

THEOREM 6. Let $|a|>1$, then

$$
\begin{aligned}
\left|\delta / a^{n}\left(|a|^{2}-1\right)^{2}\right| \leqq & \rho_{n}\left(\gamma /(z-a)+\delta /(z-a)^{2}\right) \\
\leqq & \left|\delta / a^{n}\left(|a|^{2}-1\right)^{2}\right| \\
& +\left|\left[\gamma+\left(\delta\left[(n+2)|a|^{2}-n\right]\right) / a\left(1-|a|^{2}\right)\right] / a^{n}\left(1-|a|^{2}\right)\right| .
\end{aligned}
$$

Again we have equality on both sides if $\gamma a\left(1-|a|^{2}\right)=-\delta\left[(n+2)|a|^{2}-n\right]$, in this case $\rho_{n}\left(\gamma /(z-a)+\delta /(z-a)^{2}\right)=\left|\delta / a^{n}\left(|a|^{2}-1\right)\right|$.

Since the proofs of Theorems 5 and 6 are almost identical we shall prove only Theorem 6 and then given an indication of how that proof can be applied to Theorem 5 . 
Proof. As with polynomials of degree $n$, there exists a unique rational function $Q_{n, a}(z)$ of the form $Q_{n, a}(z)=C /(z-a)+p_{n}(z)$, of best uniform approximation to $f(z)=\gamma /(z-a)+\delta /(z-a)^{2}$ on $U$, where $p_{n}(z)$ is a polynomial of degree $n$. In fact, $Q_{n, a}(z)$ is given by

$$
Q_{n, a}(z)=\left[\gamma(z-a)+\delta-K z^{n}(1-\bar{a} z)^{2}\right] /(z-a)^{2},
$$

where $K=\delta / a^{n}\left(1-|a|^{2}\right)^{2}$.

In order to demonstrate this we employ a method developed by T. J. Rivlin [4]. Since $\left|f(z)-Q_{n, a}(z)\right|=\left|K z^{n}(1-\bar{a} z)^{2} /(z-a)^{2}\right|=|K|$, the deviation $f(z)-Q_{n, a}(z)$ attains its maximum modulus everywhere on $U$, and thus by Kolomogorov's Theorem it is sufficient to show that for any rational function $R_{n}(z)$ of the form $R_{n}(z)=C /(z-a)+p_{n}(z)$ (where $p_{n}(z)$ is a polynomial of degree $n$ ),

$$
\left.\min _{z \in U} \operatorname{Re} R_{n}(z) \overline{\left[K z^{n}(1-\bar{a} z)^{2} /(z-a)^{2}\right.}\right] \leqq 0 .
$$

Now since $\bar{z}=1 / z$ for $z \in U$,

$$
\operatorname{Re} R_{n}(z) \overline{\left[K z^{n}(1-\bar{a} z)^{2} /(z-a)^{2}\right]}=\operatorname{Re} R_{n}(z)\left[\bar{K} z^{-n}(z-a)^{2} /(1-\bar{a} z)^{2}\right]
$$

is harmonic in the extended region $|z|>1$ and has a zero (at $a$ ) there, so by the minimum modulus principle for harmonic functions, (6.1) must hold.

To obtain the left side of our equality, we note that the set of polynomials of degree $n$ is included in the set of rational functions of the form $C /(z-a)+p_{n}(z)$, and so $\left\|f-Q_{n, a}\right\|_{U}=|K|=\left|\delta / a^{n}\left(1-|a|^{2}\right)\right| \leqq \rho_{n}(f)$.

To arrive at the right inequality we note that $Q_{n, a}(z)=C_{0} /(z-a)+q_{n}(z)$, where $C_{0}=\gamma+\delta\left[(n+2)|a|^{2}-n\right] / a\left(1-|a|^{2}\right)$ and $q_{n}(z)$ is a polynomial of degree $n$. Thus $\rho_{n}(f) \leqq\left\|f-Q_{n, a}\right\|_{U}+\rho_{n}\left(C_{0} /(z-a)\right)$. Since $\rho_{n}\left(C_{0} /(z-a)\right)=\left|C_{0} / a^{n}\left(1-|a|^{2}\right)\right|$, the righthand inequality follows.

To prove Theorem 5 we employ the same methods, approximating $f(z)$ by rational functions of the form $C /(z-b)+p_{n}(z)$, to obtain the left inequalities. The right inequalities are immediate.

7. An estimate for $\rho_{n}(1 /(x-\beta),[-2,2])$. We conclude by obtaining an estimate for a simple rational function, namely $1 /(x-\beta)$, on the interval $[-2,2]$. It should be noted, however, that this estimate can be applied to the above function on any closed bounded line segment in the plane provided of course that $\beta$ is not on that segment. Now, for $\beta \notin[-2,2]$, choose $b,|b|>1$, where $b+1 / b=\beta$. The number $b$ is uniquely determined and we have the following:

THEOREM 7. $R /|b|^{n+1} \leqq \rho_{n}(1 /(x-\beta),[-2,2]) \leqq R /|b|^{n-1}$, where

$$
R=\left|(1 / b-b)\left(1-|b|^{-2}\right)\right|^{-1} \text {. }
$$

Proof. We use the fact [3] that

$$
\rho_{n}(1 /(x-b),[-2,2])=\rho_{2 n}\left(z^{n+1} /(z-b)(z-1 / b), U\right),
$$

and then apply Theorem 5 , case (2), to arrive at our desired estimates. 


\section{REFERENCES}

1. N. I. Ahiezer, Lectures on the theory of approximation, OGIZ, Moscow, 1947; English transl., Ungar, New York, 1956. MR 10, 33; MR 20 \#1872.

2. S. Ja. Al'per, Asymptotic values of best approximation of analytic functions in a complex domain, Uspehi Mat. Nauk 14 (1959), no. 1 (85), 131-134. (Russian) MR 21 \#3577.

3. S. J. Poreda, Best approximation to some rational functions, Thesis, University of Maryland, College Park, Md., 1970.

4. T. J. Rivlin, Some explicit polynomial approximations in the complex domain, Bull. Amer. Math. Soc. 73 (1967), 467-469. MR 35 \#3068.

Clark University,

WORCESTER, MASSACHUSETTS 01610 